\title{
Análisis de la situación actual y zonificación integral del territorio de Pochocuape, Managua 2015
}

\section{Current situation and comprehensive zoning of Pochocuape locality, Managua 2015}

\section{Carmen María Flores Machado}

Dirección de Extensión, Universidad Nacional Autónoma de Nicaragua.

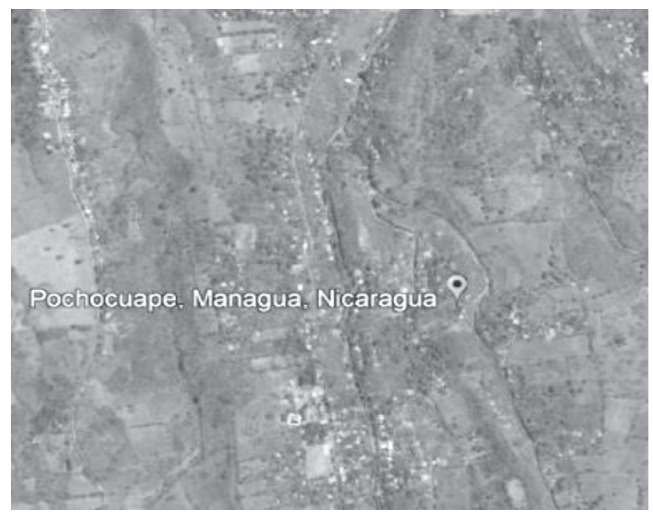

\section{RESUMEN}

En Nicaragua existen años de experiencia de planificación en los niveles territoriales, que se traducen en retos para los procesos de actuación en los territorios. La zonificación integral de un territorio tiene como finalidad la esquematización de las dinámicas territoriales, y considera que en la división del espacio existen zonas relativamente homogéneas, pero diferentes la una de la otra, en relación a las problemáticas de desarrollo. Este último aspecto puede ser utilizado para la promoción, articulación y armonización de visiones ante las problemáticas territoriales y su abordaje con los actores locales. El objetivo del presente trabajo fue analizar la situación actual del territorio de Pochocuape y zonificar integralmente la comunidad. El trabajo trasciende a un simple inventario de recursos en las diferentes dimensiones del desarrollo, y persigue entender la lógica de ubicación y distribución espacial de los mismos, así como las interrelaciones entre las dimensiones, para finalizar con la zonificación de la comunidad. El presente estudio es de carácter descriptivo y analítico, con un enfoque cuantitativo. Contó con una muestra de 230 hogares seleccionados por conveniencia, y fue realizado durante el mes de mayo del año 2015. Tomando en consideración las características particulares para la zonificación de la comarca, se identificó que pese a la cercanía a la zona urbana del distrito tres, todavía prevalece la ruralidad. Se comprobó, acorde a las dimensiones planteadas, que para el aspecto ambiental existe una sub clasificación en tres zonas, dos zonas para lo social y tres para lo económico. Lo ambiental y lo económico son coincidentes, y existe heterogeneidad en el ámbito social.

Palabras clave: desarrollo rural, territoriorialidad, dimensiones del desarrollo, zonificación integral.

\begin{abstract}
In Nicaragua there are years of planning experience at the territorial levels, which are translate into challenges for action processes at the territories. The integral zoning of a territory has the purpose of schematize the territorial dynamics, and considers that in the division of space there are relatively homogeneous zones, but different from one another in relation to development problems. The last aspect can be used for the promotion, articulation and harmonization of visions in the face of territorial problems and their approach with local actors. The objective of the present work was to analyze the current situation of the Pochocuape territory and integrally zoning the community. The work transcends a simple inventory of resources in the different dimensions of development, but seeks to understand the logic of the location and spatial distribution of them, as well as the interrelations between the dimensions, to finish with the zoning of the community. The present study was descriptive and analytical, with quantitative approach. It had a sample of 230 households selected for convenience, and was carried out during May 2015. Taking into consideration the particular characteristics for zoning the region, it was identified that despite the proximity to the urban area of district three, rurality still prevails. It was found, according to the dimensions proposed, that for environmental dimension there is a sub classification in three zones, two zones for the social dimension and three for the economic. The environmental and the economic dimensions are coincident, and there is heterogeneity in the social domain.
\end{abstract}

Key words: Rural development, territoriality, dimensions of development, integral zoning.
Recibido: 5 de diciembre 2016 Aceptado: 30 de marzo del 2017
Los artículos de la revista La Calera de la Universidad Nacional Agraria, Nicaragua, se comparten bajo términos de la licencia Creative Commons: Reconocimiento, No Comercial, Compartir Igual. Las autorizaciones adicionales a las aquí delimitadas se pueden obtener en el correo freddy.aleman@ci.una.edu.ni . Universidad Nacional Agraria 


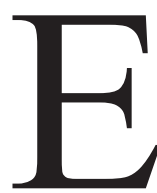
n la bibliografía se encuentran diferentes definiciones de "territorio" en el marco del desarrollo rural, alguno producido a partir del espacio geográfico, por las redes, circuitos, y flujos proyectados y materializados por los grupos sociales. El concepto de territorio desde una visión sistemática, da mucha importancia al carácter multidimensional del mismo.

La aparición de la territorialidad en el debate sobre desarrollo rural, no es casual, surge como una respuesta a los cambios económicos y sociales producidos dentro de cada país y en el contexto político de la globalización. Este responde a la necesidad de adaptar metodologías, instrumentos y actividades a los nuevos requerimientos impuestos por estos cambios e impactos no deseados. Finalmente este debate promueve una visión diferente de la problemática territorial e involucra directamente a las poblaciones en el diseño de nuevas perspectivas de desarrollo.

En Nicaragua existen años de experiencia de planificación en los diferentes niveles territoriales, estos procesos han dejado lecciones aprendidas, que se pueden traducir en retos para los procesos de planificación territorial; para superar estos, en Pochocuape se incorporaron diferentes principios y dimensiones que conllevan a identificar lo estratégico vinculando lo sectorial con lo territorial.

Para ello, se empleó el enfoque territorial del desarrollo rural, el cual promueve la articulación y armonización, en el territorio, de políticas sectoriales, fundamentada en procesos de planificación participativa con los actores sociales e institucionales y en función de una visión compartida del territorio.

El análisis territorial es un estudio realizado para identificar la diversidad de situaciones ambientales, sociales y económicas dentro de un territorio para luego analizarlas de manera integral para determinar los principales elementos que explican los paisajes y sus vínculos con los aspectos socio-culturales, políticos-institucionales, con el fin de conocer la dinámica del territorio, sus principales procesos de cambio y limitaciones para su desarrollo y por tanto prever su bienestar en beneficio de las familias que ahí habitan, como del país en general.

En este proceso se aplicó una serie de herramientas participativas e incluyentes mediante las cuales la comunidad Pochocuapeña ha caracterizado su territorio como base para la planificación territorial estratégica; entendiendo la planificación estratégica como el proceso que permite identificar, priorizar y registrar dichas metas de forma tal que ayuden a orientar el accionar de la sociedad.

El estudio tiene el objetivo de analizar la situación actual del territorio de Pochocuape y zonificación integral de la comunidad, donde más que hacer un simple inventario de los recursos en las diferentes dimensiones del desarrollo -ambiental, económico, social, político-institucional y cultural-, persigue entender la lógica de la ubicación y distribución espacial de los mismos, así como las interrelaciones entre las dimensiones, finalizando con la zonificación de la comunidad para posteriormente facilitar la identificación de dinámicas territoriales claves sobre las cuales incidir para el desarrollo de la comunidad.
Este tema es de relevancia nacional por la aplicación de la metodología del diagnóstico territorial integral, herramienta de la planificación territorial de la comunidad, que permite realizar un análisis prospectivo desembocado en la generación de escenarios de cómo puede ser el futuro del territorio.

\section{MATERIALES Y MÉTODOS}

Tipo de investigación. El estudio es de tipo analítico descriptivo sobre las interrelaciones de las dimensiones de desarrollo de la comunidad de Pochocuape. En el que se utilizó la herramienta del Diagnóstico Territorial Integral (DTI) en el que se integra el análisis de la estructuración espacial del territorio que desemboca en la zonificación integral del mismo, entendiéndose zonificar, como la división del espacio en zonas relativamente homogéneas pero diferentes en relación a su problemática del desarrollo.

Área de estudio. La comunidad de Pochocuape se encuentra ubicada en las Sierras de Managua, en el distrito III, la cual se localiza en la parte sur occidente de la ciudad de Managua. Gran parte de su territorio se encuentra en la cuenca de Las Sierras de Managua; aproximadamente el 60\% de su territorio es rural y el resto urbano.

Geográficamente limita al norte con el distrito II, al sur con el municipio de El Crucero, al este con el distrito IV y distrito V, al oeste con el municipio de Sandino. Por su extensión es uno de los distritos más grandes del municipio de Managua.

El distrito III está conformado por una parte que es urbana y tienen en su territorio las comarcas de Nejapa, Chiguilistagua, Cedro Galán, San José de la Cañada, Pochocuape, San Isidro Libertador y Ticomo.

El Universo fue de 490 hogares con una muestra de 230 familias, que fueron entrevistadas para conocer sobre cada una de las dimensiones del desarrollo, esta incluía a 23 personas entre líderes comunitarios y productores necesarias para complementar las observaciones del paisaje, aportando elementos de interpretación del mismo a partir de la experiencia y conocimiento de los habitantes (Alcaldía de Managua, 2005).

Metodología. En conformidad con esta visión sistémica del territorio, la propuesta metodológica para el análisis de la situación actual del territorio de Pochocuape, parte del análisis de la situación actual del territorio, donde se analizó primero sus diferentes dimensiones vinculado con la estructuración espacial del territorio, luego sus tendencias históricas y dinámicas de cambio, entrelazando las diferentes dimensiones, para luego integrar y validar los resultados desde un interpretación multidimensional del territorio. Se empleó entrevistas a informantes claves como líderes comunitarios, productores e instituciones públicas. Se encuesto a los jefes de familias. La metodología se puede sintetizar en los siguientes pasos: a) se realizó una revisión y estudios de documentos del territorio que nos permitiese elaborar las primeras hipótesis sobre posibles factores de incidencia en la localización de los capitales territoriales en el territorio; b) observación e interpretación de los paisajes mediante recorridos de campo 
sobre la base de las observaciones cartográficas de las principales heterogeneidad de la comunidades para la identificación de los principales ecosistemas, y modos de explotación de los mismos en base a la observación del aspecto global de los ecosistemas artificializados, así como, los capitales territoriales, y obtener elementos para entender la lógica de la ubicación de los capitales territoriales del territorio Pochocuape; c) construcción de perfiles explicativos. La información obtenida con los recorridos permitió verificar y consolidar las hipótesis realizadas anteriormente, así como, realizar una zonificación preliminar del territorio. Para lo cual se analizó y sintetizó los resultados, por medio de la realización de perfiles y/o esquemas explicativos del territorio. El diseño de estos perfiles es útiles para identificar como se distribuyen las poblaciones, la economía y el ambiente.

Los perfiles explicativos son representaciones esquemáticas que integran, de forma resumida, sobre un perfil o esquema reflejando la heterogeneidad de la región de estudio, las principales informaciones recolectadas. Este tipo de gráficos nos permitirá integrar y representar muchos datos de forma bien sintética y explicativa. Su elaboración se realizó tomando los criterios de altura y orientación para la realización de los trazos del perfil; d) las dinámicas territoriales / procesos de cambio territorial, además de aprovechar cada uno de los pasos anteriores para entender el funcionamiento del territorio, durante los recorridos de campo y visitas a las diferentes organizaciones, se aprovecha para la realización de entrevistas complementarias.

Se utilizó la realización de entrevistas complementarias con el objetivo fundamental de complementar las observaciones del paisaje aportando elementos de interpretación del mismo a partir de la experiencia y conocimiento de los habitantes. Esta se debe concebir como un intercambio donde el investigador/facilitador comparte sus observaciones y las confronta con la experiencia de los entrevistados para poder explicarlas. Estas entrevistas se realizaron a lo largo del recorrido, con base a una guía de reflexión.

Una forma de asegurar la calidad de la información recolectada, fue la comprobación con la misma entrevista a otros actores, para tener un análisis cruzado de todas estas aportaciones que permitiera tener una mejor apreciación de lo que sucede en los lugares que se recorren.

\section{RESULTADOS}

Sexo y edad del jefe de hogar. En el 53.5\% (123 hogares) el jefe de familia era del sexo femenino y el 46.5\% (107 hogares) del sexo masculino, siendo el rango de edad predominante entre 34 y 48 años en ambos sexos representando el $39 \%$, seguido del grupo de edad entre 19 y 33 años $(28.7 \%)$ y entre 49 y 63 con $22.6 \%$. La población mayor de los 64 años represento menos del $10 \%$.

Sexo de jefes de hogar y años de habitar en la comunidad. El 57\% de los jefes de hogares tienen de vivir más de 20 años en la comunidad, siendo los del femenino los que tienen más tiempo de habitar en el territorio, sobresaliendo el jefe masculino para el grupo de menos de diez años.
Nivel de escolaridad según sexo de jefes de hogares. El nivel de escolaridad es mayor en las mujeres que en los varones; un $23.67 \%$ lograron culminar la educación secundaria mientras que los varones registran un $19.81 \%$. Igualmente el nivel de educación superior es mayor en las mujeres que en los varones, sin embargo, los porcentajes son bajos, 4.83 para las mujeres y 1.93 para los hombres.

Otros aspectos socioeconómicos. El ocho por ciento de los jefes de hogares no tienen vivienda propia sino que es alquilada o prestada, siendo más acentuada esta condición en los varones que las mujeres. Esto muestra que la mayoría de los hogares tienen como activos físicos su vivienda y el terreno, lo que contribuye con el bienestar de las familias.

Los hogares de la comunidad Pochocuape utilizan tres recursos para cocinar, leña (53\%) y gas butano (57\%), y en tercer lugar la electricidad. Esto demuestra un uso habitual $\mathrm{y}$ tradicional de la leña, lo que se traduce en una sobrecarga de los recursos locales y el ambiente.

La dieta habitual de las familias incluye grupo de azucares, más del $90 \%$, seguido de los grupos vegetales, frutas, farináceos, leguminosas y grasas con más del $80 \%$; el grupo de carnes y leche con menos del $70 \%$. Las formas de preparación (fritas y cocida) registran un 58\% y asados menos del $20 \%$.

El $21 \%$ de los hogares realizan menos tres tiempos de comida al día y el $77 \%$ de ellos entre tres a cuatro tiempos, observándose que un cuarto de los hogares tienen problema en el acceso de los alimentos que repercute negativamente en la a calidad de la alimentación de las familias.

Ambientalmente el territorio de Pochocuape se divide en tres, considerando los criterios de relieve, suelo y vegetación, se determina: Una primera zona nombrada de poca vegetación, se ubica hacia el norte del distrito III del municipio de Managua. Con topografía regular, altura superior a los $100 \mathrm{msnm}$, con relieve semiplano y pendiente suave; el suelo es franco arenoso y se localizan algunos árboles frutales como cítricos y musáceas, además granos básicos como frijol y maíz.

Una segunda zona denominada de vegetación arbustiva abarca una extensión de dos y medio kilómetros de largo, la parte media del territorio cuenta con un relieve irregular con planicies y pequeñas cuestas con pendientes de 0 a $15 \%$.

El suelo es arenoso y con vegetación arbustiva. El paisaje de esta zona presenta trechos de vegetación y despales, las familias se dedican a cultivar hortalizas y granos básicos.

Una tercera zona llamada escarpada con árboles dispersos se localiza hacia el sureste de la meseta de las sierras de Managua, esta zona presenta diferentes elevaciones con más $400 \mathrm{msnm}$, es la más alta de la comarca y presenta un relieve que comprende planicies afectadas con cárcavas y cuestas. 


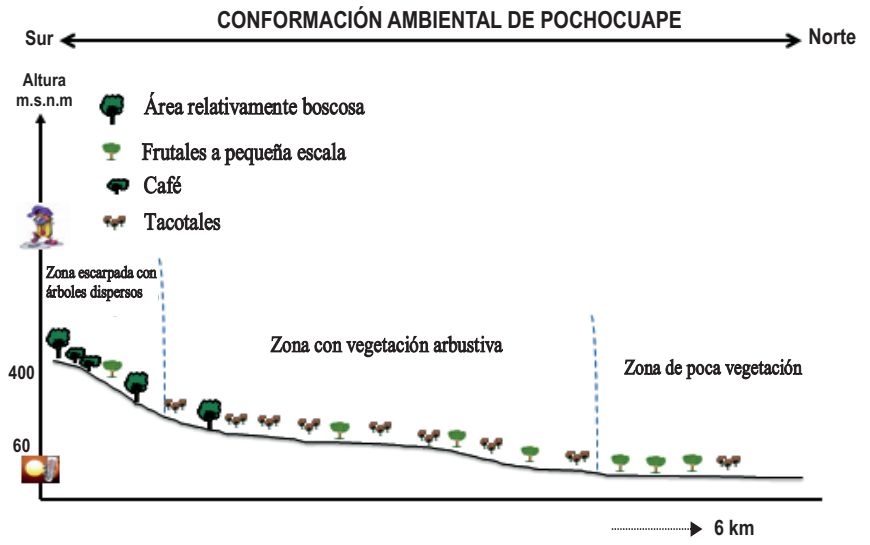

Figura 1. Caracterización ambiental de la comunidad de Pochocuape, 2015.

En base a criterios de análisis de equipamiento, infraestructura, patrimonio histórico y patrimonio arquitectónico, procesos y mecanismos de acceso a los factores productivos, recursos financiero, tenencia de la tierra, otros medios de producción, actividades y empresas, concentración geográfica y estructuración de las empresas y de los sectores productivos de la economías y mercados y relaciones externas, integración de los mercados, redes de intercambios y procesos de comercialización, se diferencia tres zonas en la dimensión económica.

Zona de dinámica económica y trabajo local. Denominado así, por su comercio interno y la fuerza de trabajo que se transporta diariamente a la ciudad de Managua, entre el comercio existente en la zona sobre salen pulpería, venta de cosmético, dulcería, venta de productos de frutas y verduras, entre otros. En general trabajan en mercados, zona franca en su mayoría y empresas comerciales, construcción, ventas ambulantes y moto taxis. En esta zona los caminos son buenos pavimentados, con circulación de transporte urbano y de comercio que permite el fácil acceso a los productos básicos de las familias.

Segunda zona de agricultura familiar. Esta zona está ubicada más al sur este de la comarca con caminos buenos en épocas de veranos, no así en el invierno que se hace poco accesible por las correntadas de agua que atraviesan por el mismo por ser cauces naturales del agua que bajan desde El Crucero y recorren la comunidad hasta el Lago de Managua.

Tercera zona agroforestal. Esta zona debido a las condiciones de altura, precipitación, suelos y temperatura, se establecen plantaciones de café en sistemas agroforestales, existiendo fincas dedicadas a este rubro, además de otros como granos y hortalizas.

Con respecto a las dimensiones social-humana y político-institucional de la comunidad de Pochocuape, se diferencia dos zonas:

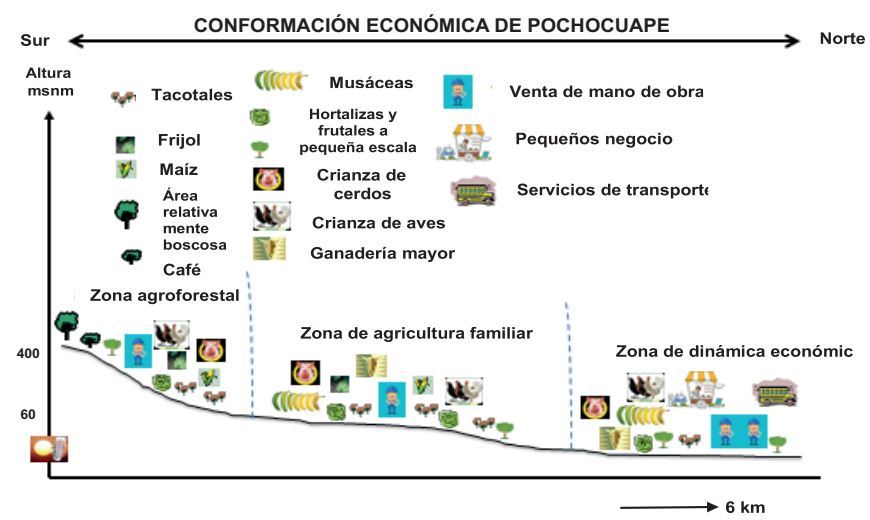

Figura 2. Caracterización económica de la comunidad de Pochocuape, 2015.

Zona denominada "Concentración". Coincide con la zona de comercio identificada desde el punto de vista económico. En esta zona la carretera ha sido un detonante para el establecimiento de caseríos y pequeños negocios, lo que ha dinamizado no solo la economía, sino las relaciones sociales (cooperativas, religiosas y políticas), actores sociales presente del gobierno local. Con acceso a servicios básicos.

Zona llamada "Dipersa". Se encuentra en la parte más alta de la subcuenca sur de Managua. Se compone del mayor número del territorio de la comunidad. Aunque, en los últimos años, se han mejorado los servicios y relaciones, estas todavía no son óptimas para el fortalecimiento del capital humano. Si bien, las poblaciones acceden a bienes y servicios establecidos en la comunidad, estos son limitados ya que las distancias y el estado de los caminos limitan el acceso de las familias que habitan en la zona más alta. Las redes sociales también son limitadas; la mayoría no pertenecen a ninguna organización, principalmente productivas, que le permitan tener mayor oportunidad de acceder a financiamiento para la producción agrícola.

El acceso de los servicios básicos es limitado principalmente de salud y educación; no existe infraestructura en esta zona solo 'por el organismo Samaritan Internacional que atiende a 300 escolares y brinda atención de salud y de alimentación.

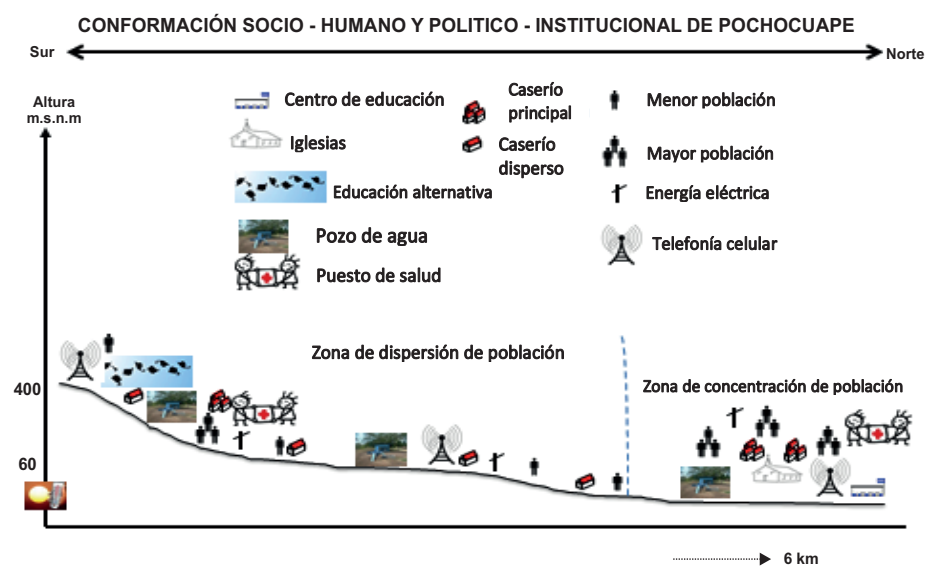

Figura 3. Caracterización humana y político-institucional de la comunidad Pochocuape, 2015. 
En la estructuración actual del territorio de Pochocuapes, producto de las interrelaciones de las dimensiones del desarrollo, se identificaron tres zonas diferenciadas por sus potencialidades y limitaciones en las dimensiones económicas, sociales, ambientales y político institucionales. Una zona I llamada Dinámica Económica y mayor Desarrollo Humano, cuenta con servicios básicos, con vías de acceso a transporte, con infraestructura de educación, salud y mayor dinamismo económico local dedicado en su mayoría al comercio y la fuerza de trabajo en los sectores aledaños a la capital. La zona II denominada Agricultura Familiar y Degradación Ambiental, trabaja más en la producción de frijoles, maíz, plátanos, tomates y hortalizas en pequeñas parcelas dos veces al año y presenta problemas de abastecimiento de agua en la época seca. La Zona III nombrada Alta con presencia de Sistema Agroforestal con Asistencia Social, está ubicada en la parte más alta de la comunidad y se dedica a la agricultura familiar en presencia de algunos sistemas agroforestales, principalmente café, estos sistemas están en manos de diez pequeños productores con parcela entre dos y tres manzanas $(1.41-2.11 \mathrm{ha})$, con escases de agua en época seca y con poca crianza de animales de especies menores. La fertilización de los cultivos es a base de fertilizantes químicos, lo que ocasiona contaminación en los suelos.

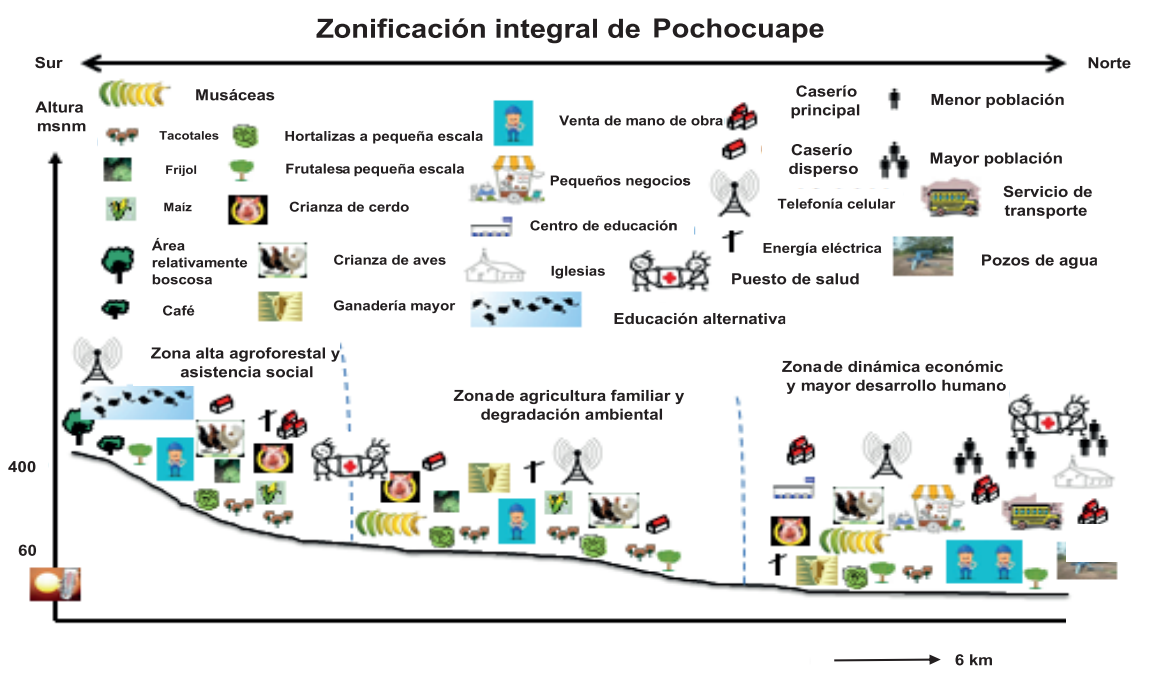

Figura 4. Zonificación integral de la comunidad de Pochocuape, 2015.

\section{DISCUSIÓN}

Más de la mitad de los jefes de hogares son mujeres y en el caso de los varones esto representa el $46 \%$; el rango predominante es menor de 49 años (67.7\%), sobresaliendo el rango de edad entre 34 y 48 años en ambos sexo con casi el $40 \%$. Estos datos son coincidentes con la estadística nacional que indica una población nicaragüense relativamente joven (INIDE, 2015). Esta característica demográfica es importante porque los hogares están formados por familias jóvenes con aportación significativa en la vida laboral y contribución a la economía familiar y de la comunidad.
La mayoría de las familias con vivienda propia, utilizan leña para cocinar los alimentos, con garantía de una alimentación adecuada con más de tres tiempo al día, y con una variedad de alimentos de siete grupos entre azucares, vegetales, frutas, farináceos, leguminosas, grasas, carnes y leche, siendo la forma más común de preparación de los alimentos fritas y cocida. Cualitativamente la alimentación es balanceada ya que integra alimentos de origen animal y vegetales, fuente de nutrientes esenciales (FAO, 2007).

La dimensión socio-humano, se manifestó con una marcada dinámica poblacional, siendo la mayoría del lugar con tierras de herencia de sus padres; un pequeño grupo de pobladores son buscados para el cuidado de las fincas que aún existen en el territorio a cargo de productores individuales. Existe un cierto grado de especulación en tierras, principalmente en las zonas altas por el atractivo del paisaje y el clima. Actualmente la población joven ha perdido el interés por trabajar la tierra como lo hacían antes donde cultivaban café, frijol y maíz. Existen algunos núcleos productivos a cargo de mujeres, ya que las cooperativas han dejado de funcionar limitando el acceso de los pequeños productores a financiamiento y tecnificación para la producción de alimentos hortalizas. La organización comunitaria es baja, solo el 11\% de los pobladores pertenecen a alguna organización comunitaria. La comarca cuenta con servicios básicos: $70 \%$ de la población con acceso todo el año a agua, el resto la compran, principalmente en época de verano. Se cuenta con servicio de energía casi en su totalidad, servicios higiénicos, principalmente letrinas. No hay servicios de agua negras. Cuenta con un centro educativo de pre- escolar, primario y secundario a distancia, con un puesto de salud que atiende tres días a la semana, tres iglesias, un cementerio. También tienen una carretera pavimentada con trechos deteriorados y anegados en la época lluviosa. En la dimensión ambiental se cuenta con tres zonas: poca vegetación, vegetación arbustiva y escarpada con árboles dispersos; en la dimensión económica encontramos dinámica económica y trabajo local, agricultura familiar y agroforestal. Desde la dimensión social-humana y político- institucional, en la comunidad se diferencia dos zonas: la denominada concentrada y la llamada dispersa.

La actividad económica de la comunidad en el área rural es agropecuaria, los principales cultivos son frijol, maíz -de autoconsumo- y sorgo rojo destinado para la alimentación animal. En el área urbana la actividad es principalmente la industria y el consumo, esto debido al crecimiento urbanístico.

Esta zona ha perdido su vocación agrícola producto de la migración de pobladores, por la deforestación y la erosión del suelo la cual no ha sido atendida adecuadamente por la falta de asistencia técnica y capacitaciones que ayude a conservar el agua y los suelos y muy pocos hacen medidas de conservación de estos recursos a pesar que no cuentan con la suficiente tecnología. 
La zonificación integral del territorio en cada una de las dimensiones del desarrollo sostenible demarcado en tres zonas: Zona I llamada Dinámica Económica y mayor Desarrollo Humano con las principales características en la zona de recarga hídrica con abundante fuentes superficiales y la zona con mayor actividad de servicio y comercio. La Zona II: designada Agricultura Familiar y Degradación Ambiental que se caracteriza por contar limitado recursos naturales, recursos hídricos con limitado acceso en época de verano y suelos francos favorecidos por la sedimentación de las zonas más alta, sumado a las condiciones agroclimáticas permiten siembras tradicionales y la siembra de apante. La Zona III: nombrada Alta con presencia de Sistema Agroforestal con Asistencia Social caracterizada por escasas áreas compactas de bosque, lo que implica menor diversidad de especies de flora y fauna autóctona en relación a las zona 1 y 2; las familias productoras están utilizando algunas acciones orientadas a mantener efectos benéficos de los árboles en las unidades producción, mayor presencia de organizaciones gubernamentales y organismos no gubernamentales en la zona 1 y 2 fomentando acciones orientadas a promover la igualdad de género y la pequeña producción agrícola complementa su ingreso con la venta de mano de obra temporal y / o migración interna o externa.

Los procesos centrales que cambian la estructuración actual y actores sociales de la comunidad de Pochocuape sirven para entender la lógica de la ubicación de los capitales territoriales sobre sus trayectorias de evolución históricas en base al análisis de la evolución y diferenciación de las áreas identificadas, en la dimensión económica (Dinámica en el Sistema Productivo y la Urbanización), en lo ambiental (la Pérdida de la cobertura boscosa) y en lo socio-humano político institucional (Aumento de la Población).

\section{CONCLUSIONES}

La mayoría de los jefes de hogares tienen más de 20 años de vivir en la comunidad, siendo en su mayoría del sexo femenino, esto demuestra que los hogares en su mayoría carece de la figura paterna y existe una sobrecarga de responsabilidad en la mujer tanto en crianza como en el sustento de los hijos.
El nivel de educación secundaria y superior de los jefes de hogares es mayor en las mujeres que los varones, esto es un factor protector para los hogares por el rol de las mujeres en el aprovechamiento de los recursos económicos.

El contexto socio-humano de la comunidad, se encuentra provista de servicios básicos de agua, luz eléctrica, telefonía, transporte, caminos en regular estado, centro de educación, salud y comunicación, iglesias y cementerio.

La estructura económica está constituida por una agricultura de subsistencia y comercio. Entre las actividades sobresalen la producción de granos básicos y hortalizas a pequeñas escala y las actividades de comercio y servicio, bajando a los barrios aledaños, empresas y otros sectores productivos de la capital.

En el contexto ambiental la comunidad presenta serios problemas de deforestación en la parte sur de su territorio, y de erosión severa existente en la cuenca por la eliminación de los bosques tupidos en la zona de las Sierras de Managua debido al avance de la frontera agrícola y al crecimiento urbanístico desordenado que limita la infiltración y causan inundaciones cada vez más grave en la comunidad.

En la dimensión ambiental se registran una zona de poca vegetación, otra de vegetación arbustiva y una tercera escarpada con árboles dispersos; económicamente se clasifican como dinámica económica y trabajo local, agricultura familiar y agroforestal. Desde la dimensión social-humana y político-institucional, en la comunidad se denomina una zona concentrada y una zona dispersa.

En la zonificación integral del territorio en cada una de las dimensiones del desarrollo sostenible se demarcan tres zonas: zona I llamada dinámica económica y mayor desarrollo humano, la zona II: designada agricultura familiar y degradación ambiental y la zona III nombrada alta con presencia de sistema agroforestal con asistencia social.

Los procesos de cambios más marcados en la comunidad son cuatro, en la dimensión económica (dinámica en el sistema productivo y la urbanización), en lo ambiental (la pérdida de la cobertura boscosa) y en lo social (aumento de la población).

\section{REFERENCIAS BIBLIOGRÁFICAS}

Alcaldía de Managua. 2005. Caracterización del distrito III de Managua. Mangua, NI.

FAO (Organización de las Naciones Unidas para Alimentación y la Agricultura, IT). 2007. Guía de Seguridad Alimentaria y Nutricional para el uso del personal agropecuario de Nicaragua. Managua, NI.

INIDE (Instituto Nacional de Información de Desarrollo, NI). 2015. Anuario estadístico 2016. (en línea). Disponible en http://www. inide.gob.ni/Anuarios/Anuario\%20Estadistico\%202015.pdf 